\title{
Dual Dirac cones in elastic Lieb-like lattice metamaterials
}

\author{
Cite as: Appl. Phys. Lett. 114, 081906 (2019); doi: 10.1063/1.5085782 \\ Submitted: 14 December 2018 - Accepted: 1 February 2019 . \\ Published Online: 28 February 2019

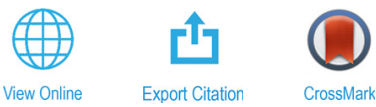

Bing Li, ${ }^{1,2}$ (D) Zheng Li, ${ }^{3}$ Johan Christensen, ${ }^{4}$ and K. T. Tan ${ }^{2, a)}$ (D)

\begin{abstract}
AFFILIATIONS
'School of Aeronautics, Northwestern Polytechnical University, Xi'an, Shaanxi 710072, China

${ }^{2}$ Department of Mechanical Engineering, The University of Akron, Akron, Ohio 44325-3903, USA

${ }^{3}$ Department of Mechanics and Engineering Science, Peking University, Beijing 100871, China

${ }^{4}$ Instituto Gregorio Millan Barbany, Universidad Carlos III de Madrid, ES-28916 Legans (Madrid), Spain
\end{abstract}

a) Author to whom correspondence should be addressed: ktan@uakron.edu

\begin{abstract}
Double-zero-index properties of electromagnetic and acoustic waveguides have been recently realized based on Dirac/Diraclike cones at the Brillouin zone (BZ) center. However, very limited research has been devoted to double-zero-index structural systems of elastic waveguides, and almost no lattice system has been able to achieve multiple separated Dirac cones generated around different frequencies at the BZ center. Here, we report two separated elastic-wave Dirac-like cones, which are simultaneously achieved around different Dirac points at the BZ center, due to the accidental degeneracy and frequency repulsion effect in a Lieb-like lattice metamaterial. Using the proposed elastic medium, the double-zero-index properties of various elastic wave modes are theoretically analyzed, numerically computed, and experimentally observed at the neighborhood of both Dirac-like points. The performance of near total transmission without the phase change and the ability of wave-front shaping are unambiguously verified by numerical simulation and experimental measurements.
\end{abstract}

Published under license by AIP Publishing. https://doi.org/10.1063/1.5085782

A rich variety of distinctive phenomena not typically found in nature have been revealed by both quantum and classical transports in crystals and metamaterials, depending on the lattice topology, interparticle interactions, presence of disorders, and external energy fields. ${ }^{1-15}$ As a topic of particular interest, flatband systems with a Dirac cone, which can generate dispersionless and macroscopically degenerated energy bands, have attracted ever-increasing attention. ${ }^{16-22}$ As one of the simplest platforms in the family of optical flat-band lattice systems, the Lieb lattice is a line-centered square depleted lattice, characterized by three atoms in one unit cell. Based on this exotic pattern, a dispersionless flat-band intersects with two linearly dispersing bands at a degenerate Dirac point, exhibiting a Dirac-like cone in the middle of the energy spectrum of the structure. ${ }^{2,18,21,22}$ The flat-band properties around the Dirac-like cone have been observed in the Lieb optical lattice and photonic crystals. Nevertheless, little research has been performed on the phononic analog of the Lieb-like lattice, despite great interest in investigating Dirac/Dirac-like cones in phononic (acoustic) counterparts.

Based on the mechanism of Bragg scattering or local resonance, there are various designs of phononic crystals and acoustic metamaterials to obtain Dirac-like cones in classical wave systems. Acoustic Dirac-like cones can be categorized into three ways: (1) A single Dirac cone composed of two linearly touching bands at the corner of the Brillouin zone (BZ); ${ }^{23,24}$ (2) A Dirac cone derived from an accidental threefold degeneracy of two linearly dispersing bands and an additional flat-band, where the Dirac point appears at the center of $\mathrm{BZ}(\Gamma$ point $) ;{ }^{25-30}(3)$ An emerging double-Dirac cone with a fourfold degeneracy, where a pair of identical Dirac cones overlap at a single Dirac point at the BZ center. ${ }^{31-35}$ Recent demonstration of Dirac/ Dirac-like cones at the center of BZ possesses enormous potential to realize the double-zero-index properties of electromagnetic (epsilon-and-mu-near-zero) ${ }^{25,36,37}$ and acoustic (concurrent zero effective density and infinite bulk modulus $^{26-30}$ ) waveguides, for exciting applications like perfect tunneling, wave-front shaping, acoustic beam collimation, and asymmetric transmission. ${ }^{26-28,30}$

Compared to electromagnetic and acoustic waves, the extension of zero-index structural systems to elastic waveguides remains an open challenge since elastic waves in solid structures have more polarization degrees of freedom, various deformation 
modes, and scattering complexity. ${ }^{38,39}$ Recently, Zhu and Semperlotti ${ }^{40}$ have reported the experimental realization of a double-zero-index elastic phononic waveguide to achieve the corresponding cloaking and supercoupling effects. However, in all these reported acoustic/elastic artificial structures with periodic unit cells, only one among the three Dirac/Dirac-like cone types is obtained by each design. Almost no lattice system has been reported to achieve dual or multiple separated Dirac cones generated around different Dirac points.

In this work, we present the development of an elastic Lieb-like lattice metamaterial to realize two separated Diraclike cones at the BZ center. The double-zero-index effective properties of various elastic wave modes around both Dirac-like points are theoretically, numerically, and experimentally investigated. A schematic of the proposed elastic Lieb-like lattice metamaterial is illustrated in Fig. 1(a). Here, the three "atoms" with sites $\mathrm{A}, \mathrm{B}$, and $\mathrm{C}$ in an optical/photonic Lieb lattice are replaced by three geometric inhomogeneities with nonperforated taper holes, symmetrical about the upper and lower surfaces. For an infinite structural waveguide, it can be further described by a unit cell [Fig. 1(b)], with a periodic array of square $\mathrm{C}_{4 v}$ symmetry.

The material properties of aluminum are applied to the proposed elastic metamaterial, where Young's modulus $\mathrm{E}=70 \mathrm{GPa}$, Poisson's ratio $\nu=0.33$, and density $\rho=2700 \mathrm{~kg} / \mathrm{m}^{3}$. Based on the Bloch-Floquet theory, we use finite element software, Comsol Multiphysics, to evaluate the dispersion relations for an infinite elastic Lieb-like lattice. The geometric parameters of a unit cell $(r=0.0183 \mathrm{~m}, a=0.00367 \mathrm{~m}, \mathrm{~L}=0.0497 \mathrm{~m}$, and $h=0.008 \mathrm{~m}$ ) yield an exciting dispersion spectrum that exhibits two Dirac-like points at frequencies of $f_{D 1}=6.27 \mathrm{kHz}$ and $f_{\mathrm{D} 2}=29.24 \mathrm{kHz}$ [Fig. 1(c)], formed at the BZ center ( $\Gamma$ point). Figures 1(d) and 1(e) show the three-dimensional dispersion surfaces around Dirac-like points I and II, respectively. As shown in Fig. 1(c), it appears that Dirac point I is a double-Dirac cone induced by a fourfold degeneracy, while Dirac point II is formed by a threefold degeneracy. However, the close-up view in Fig. 1(d) shows that Dirac point I is actually resulted from a threefold degeneracy since only three bands intersect at the Dirac point. ${ }^{31-35}$ Interestingly, if the three branches are linearly dispersive along the $\mathrm{M} \Gamma$ direction [Fig. 1(d)], then two of them shift to near dispersionless bands along the $\Gamma \mathrm{X}$ direction. The close-up view in Fig. 1(e) shows that Dirac point II is a typical threefold degeneracy of a flat-band with two linearly dispersing bands. The three relevant degenerate Bloch states at the two separated Dirac-like points are further captured and exhibited in Figs. 2(a) and 2(b), respectively.

It is observed that two kinds of mode shapes exist for each Dirac-like point: dipolar and quadrupolar field patterns. For Diraclike point I, two kinds of degenerate Bloch states are formed by the whole unit cell [Fig. 2(a)], while for Dirac point II, the Bloch states are also induced by the whole microstructure, but mainly localized in the central "atom" of the unit cell [Fig. 2(b)]. By altering the transverse radius of the semi-elliptic holes (from $r=0.0183$ to $r=0.0188$ ), the frequencies of Dirac-like cones have a slight shift, and the quadrupolar mode shape is separated from the two dipolar states [Figs. 1(d) and 1(e)]. This is indicative that both Dirac-like
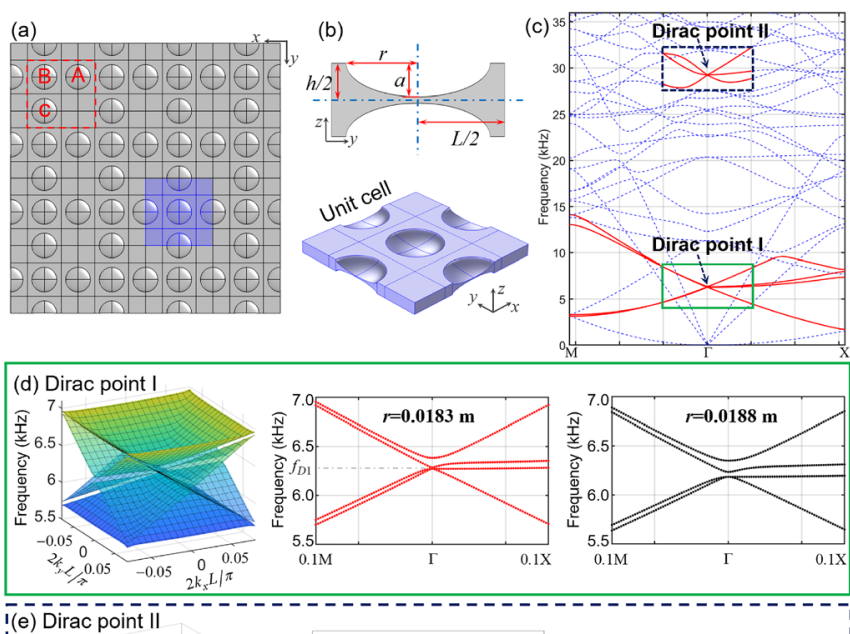

(e) Dirac point II
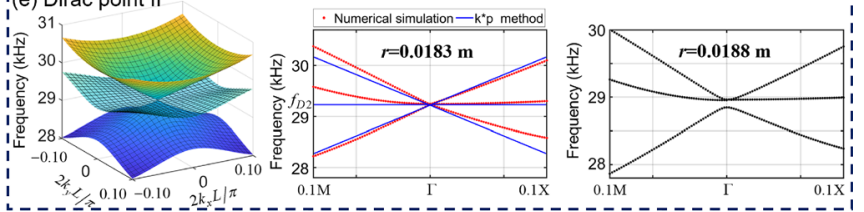

FIG. 1. (a) Schematic of the proposed Lieb-like lattice elastic metamaterial. (b) Cross-sectional and isometric view of a periodic unit cell. (c) Dispersion relations of an infinite Lieb-like lattice metamaterial with two Dirac-like points at different frequencies simultaneously obtained at the BZ center. (d) Three-dimensional dispersion surfaces around Dirac-like point I and (e) Dirac-like point II showing the formation of Dirac-like cones. The right image shows enlarged views of the threefold degeneracy, closed when $r=0.0183 \mathrm{~m}$ and reopen when $r=0.0188 \mathrm{~m}$.

points are induced by zone folding of accidental degeneracy of two dipolar states and a quadrupolar state.

To theoretically analyze the dual Dirac cones, we apply the boundary effective medium theory (BEMT) and the $\vec{k} \cdot \vec{p}$ perturbation method. On the basis of the BEMT, ${ }^{41}$ the Lieb-like unit cell can be treated as an effective homogeneous medium stimulated by the incident waves, and the equivalent material parameters of the medium can be evaluated by calculating the eigenstate fields on the boundaries. The applicability of the BEMT has been verified in elastic metamaterial waveguides, ${ }^{40,42,43}$ even at a relatively high frequency or a relatively short-wavelength. Using this method, we calculate the eigenstates of the proposed unit cell around the two Dirac points along the $\Gamma-\mathrm{X}$ direction. Around Dirac-like point I, the wavelength is about 10L (A0 mode), while around Dirac-like point II, the wavelength is about 3.41L (S0 mode). The relevant boundary responses, including the stress, strain, and displacement field distributions, are captured. Then, we use Newton's second law to calculate the effective mass density and use the constitutive relations to obtain the effective moduli. The relevant equation for the effective mass, $\rho$ eff, can be written as ${ }^{40,41}$

$$
\rho^{e f f}=\frac{m^{e f f}}{L^{2} h}=\frac{F_{z}^{e f f}}{L^{2} h \ddot{u}_{z}^{e f f}}=-\frac{F_{z}^{e f f}}{\omega^{2} L^{2} h u_{z}^{e f f}} .
$$

Here, $F_{z}^{\text {eff }}$ is the effective net force exerted on the unit cell in the $z$ direction and $u_{z}^{\text {eff }}$ is the effective displacement of the unit cell in the $z$ direction, which can be calculated as 
(a)
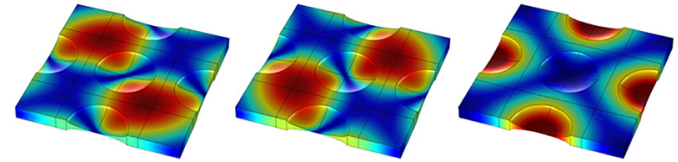

(b)
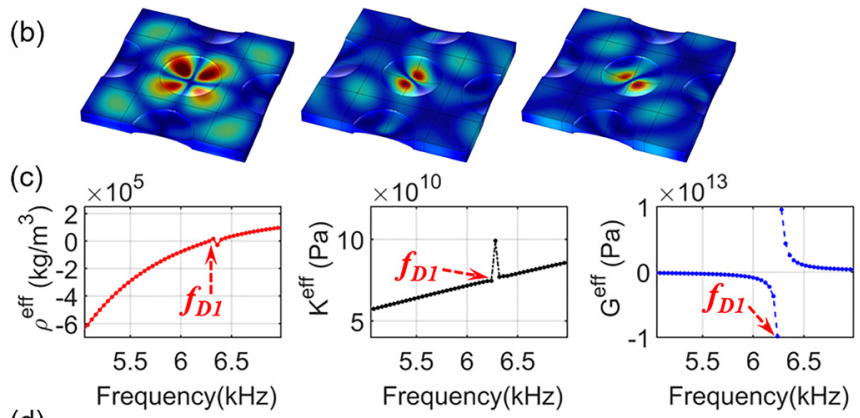

(d)

Freque
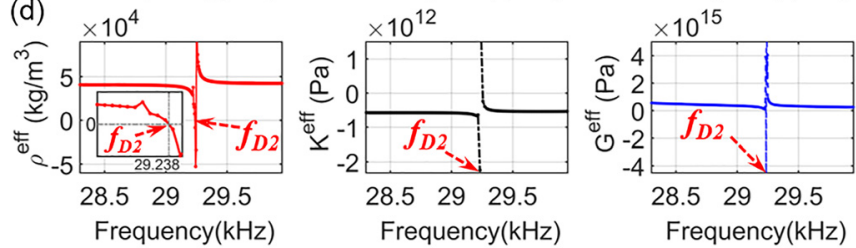

FIG. 2. Three degenerate Bloch states at (a) Dirac-like point I and (b) Dirac-like point II. The effective mass $\rho^{\text {eff }}$, effective bulk modulus $K^{\text {eff }}$, and effective shear modulus $G^{\text {eff }}$ obtained around the frequencies of (c) Dirac-like point I, $f_{D 1}$, and (d) Dirac-like point II, $f_{D 2}$. Inset: enlarged near-zero effective density-frequency profile.

$$
\begin{aligned}
\mathrm{F}_{z}^{e f f}= & \left.\iint \mathrm{T}_{x z} d y d z\right|_{x=L}-\left.\iint \mathrm{T}_{x z} d y d z\right|_{x=0}+\left.\iint \mathrm{T}_{y z} d x d z\right|_{y=L} \\
& -\left.\iint \mathrm{T}_{y z} d x d z\right|_{y=0}
\end{aligned}
$$

and

$$
u_{z}^{e f f}=\frac{\left.\iint u_{z} d y d z\right|_{x=L}+\left.\iint u_{z} d y d z\right|_{x=0}}{2 L h},
$$

where $\mathrm{T}_{x z}$ and $\mathrm{T}_{y z}$ are the $x z$ and $y z$ components of the stress tensors, respectively. The effective moduli can be calculated based on the constitutive relations as

$$
\begin{aligned}
\mathrm{T}_{x x}^{e f f} & =c_{11}^{e f f} S_{x x}^{e f f}+c_{12}^{e f f} S_{y y}^{e f f}, \quad T_{y y}^{e f f}=c_{12}^{e f f} S_{x x}^{e f f}+c_{11}^{e f f} S_{y y}^{e f f} \\
T_{x z}^{e f f} & =G_{x z}^{e f f} S_{x z}^{e f f}, \quad T_{y z}^{e f f}=G_{y z}^{e f f} S_{y z}^{e f f} .
\end{aligned}
$$

The detailed expressions of the effective stress and strain tensors are listed in the supplementary material.

According to Eqs. (1)-(4), effective density $\rho^{\text {eff }}$, effective bulk modulus $K^{\text {eff }}\left(K^{\text {eff }}=\left(c_{11}^{\text {eff }}+2 c_{12}^{\text {eff }}\right) / 3\right)$, and effective shear modulus $\mathrm{G}^{\text {eff }}$ around the frequencies of dual Dirac-like cones are calculated and plotted in Figs. 2(c) and 2(d). It is clear that at the frequency of Dirac point I $\left(f_{\mathrm{D} 1}\right)$, density $\rho^{e f f}$ is zero and shear modulus $G^{e f f}$ becomes infinite. However, the bulk modulus remains positive. Therefore, the double-zero-index property is exhibited for the antisymmetric wave mode (A0 mode) at Dirac point I. At Dirac point II $\left(f_{\mathrm{D} 2}\right)$, the proposed system shows zero effective density and infinite bulk modulus. It is expected that the double-zero-index property should be exhibited for the symmetric wave mode (S0 mode) at Dirac point II.

We further apply the $\vec{k} \cdot \vec{p}$ method to analyze the linear intersection at the Dirac-like points. The $\vec{k} \cdot \vec{p}$ method is a perturbation method originated from the field of electrons. ${ }^{44,45}$ Mei et $a .^{29}$ generalized this method to classical waves and verified its applicability in the Dirac-like cones of photonic/phononic crystals. Zhu and Sempertotti ${ }^{40}$ adapted this $\vec{k} \cdot \vec{p}$ perturbation method to analyze the degeneracy of Dirac-like cones in a bulk elastic system. Recently, Chen et al. ${ }^{46}$ and Chen et al. ${ }^{47}$ applied the $\vec{k} \cdot \vec{p}$ method to analyze the elastic topological phases in the mechanical Kagome lattice and honeycomb lattice, respectively. For an inhomogeneous thin plate consisting of Lieb-like unit cells, we can use Navier's equations to describe the elastodynamic response as

$$
\begin{aligned}
-\rho \omega^{2} \mathrm{U}= & (\lambda+\mu) \nabla(\nabla \cdot \mathrm{U})+\mu \nabla^{2} \mathrm{U}+\nabla \lambda \nabla \cdot \mathrm{U}+\nabla \mu \times \nabla \times \mathrm{U} \\
& +2(\nabla \mu \cdot \nabla) \mathrm{U},
\end{aligned}
$$

where $\rho, \lambda$ and $\mu$ are the local density and local Lame constants, $\mathrm{U}$ is the displacement vector. Based on the extended $\vec{k} \cdot \vec{p}$ method, ${ }^{29,40}$ the Bloch functions at $\vec{k}$ near $\vec{k}_{0}=0$ can be expressed by a linear combination of the $\vec{U}_{j 0}(\vec{r})$, leading to

$$
\overrightarrow{\mathrm{U}}_{n \vec{k}}(\vec{r})=\sum_{j} \mathrm{C}_{n j}(\vec{k}) e^{i \vec{k} \cdot \vec{r}} \vec{U}_{j 0}(\vec{r}),
$$

where $C_{n j}$ is the coefficient of the linear combination and " $n$ " denotes the band index. On the basis of the orthonormal relation of the basic Bloch functions $\vec{U}_{j 0}(\vec{r})$, we can further obtain the dispersion relations of the inhomogeneous plate as

$$
\operatorname{det}\left|\left(\omega_{n \vec{k}}^{2}-\omega_{j 0}^{2}\right) I+P(\vec{k})\right|=0,
$$

where $P_{j l}(\vec{k})=i \vec{k} \cdot \vec{p}_{j l}(\vec{k})$ is the reduced Hamiltonian and $\vec{p}_{j l}^{*}=\vec{p}_{l j}$. We can greatly lower the dimension of the matrix P in Eq. (7) by considering only the degenerate states at the Dirac points, which are incorporated in Eq. (6). For $\vec{k}$ close to $\vec{k}_{0}=0$, the simplified analytical solution to the dispersion relation can be written as

$$
\frac{-2 \omega_{j 0} \Delta \omega}{\Delta k}=\gamma_{\alpha}+o(\Delta k), \quad \alpha=1,2,3, \ldots
$$

Linear slopes $\gamma_{\alpha}$ can be evaluated by $\vec{p}_{j l}$. Dirac-like point II [Fig. $1(\mathrm{e})]$ is selected to verify the applicability of the extended $\vec{k} \cdot \vec{p}$ method in the proposed elastic Lieb-like metamaterial. The matrix elements of $\vec{p}_{j l}$ are numerically evaluated by using the integration of the Bloch wave functions at $\vec{k}_{0}=0$. Around the frequency of Dirac point II, we obtain $\left|\vec{p}_{12}\right|=\left|\vec{p}_{13}\right|=6.9390$ $\times 10^{8}$ (or $\left|\vec{p}_{21}\right|=\left|\vec{p}_{31}\right|=6.9390 \times 10^{8}$ ), and the other elements are zero. Consequently, the dispersion around the Dirac-like point can be written as $\Delta f / \Delta k=0$ and $\Delta f / \Delta k= \pm\left|\vec{p}_{12}\right| /\left(8 \pi^{2} f_{\mathrm{D} 2}\right)$ $= \pm 300.56$. Therefore, the first solution represents the flatband and the other two solutions are contributed by the two linearly intersecting bands at the Dirac-like point. In Fig. 1(e), the results obtained using the $\vec{k} \cdot \vec{p}$ method (blue solid lines) are 


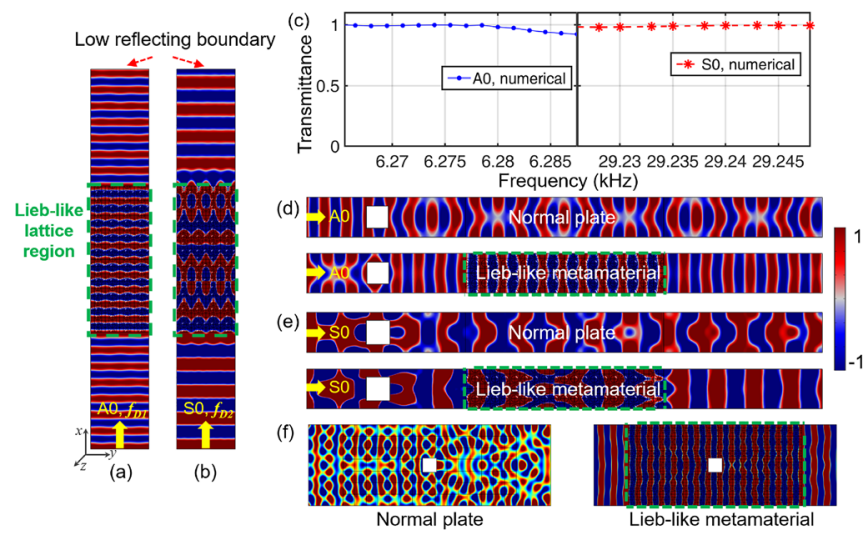

FIG. 3. (a) Out-of-plane displacement field contour $\left(u_{z}\right)$ under an incident $A 0$ planar wave at frequency $f_{D 1}(6.27 \mathrm{kHz})$. (b) In-plane displacement field contour $\left(u_{x}\right)$ under an incident S0 planar wave at frequency $f_{D 2}(29.24 \mathrm{kHz})$. (c) Numerical transmittance coefficients obtained around the two Dirac-point frequencies under $\mathrm{AO}$ and So planar waves. (d) Comparing the out-of-plane contour and (e) in-plane contour in a normal plate and a Lieb-like lattice plate, in the presence of defects. (f) Out-ofplane displacement contour under an incident $A 0$ wave at frequency $f_{D 1}$ in a normal and a Lieb-like lattice plate with a defect in the Lieb-like lattice region.

compared with the numerical dispersion relations (red dots). The good agreement verifies that the obtained Dirac-like cones are induced by the degenerated state coupling and the frequency repulsion effect.

To investigate the transmission properties of various wave modes at the frequencies of Dirac points, we numerically build an elastic aluminum waveguide consisting of a double-zeroindex Lieb-like lattice plate (middle $10 \times 4$ unit cells) [Figs. 3(a) and 3(b)]. One end (bottom surface) is excited by incident waves with different modes, and the other end is applied with a low reflecting boundary condition. Periodic boundary conditions are applied to the left and right boundaries to simulate an infinite elastic waveguide.

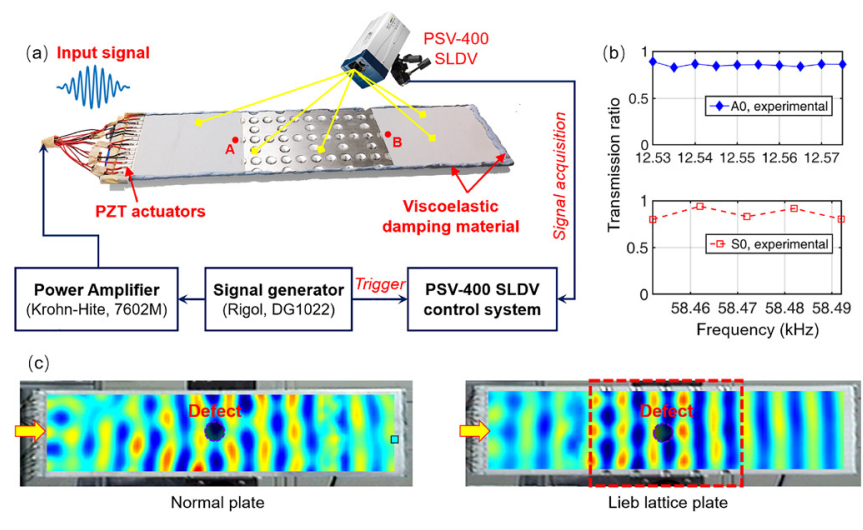

FIG. 4. (a) Experimental setup for the transmission measurement in a Lieb-like lattice waveguide. (b) Experimental transmission spectra for A0 and SO modes around the frequencies of Dirac points. (c) The out-of-plane displacement contour under $A 0$ planar wave excitation in a normal plate and a Lieb-like lattice plate with the same defect hole (diameter $14 \mathrm{~mm}$ ).

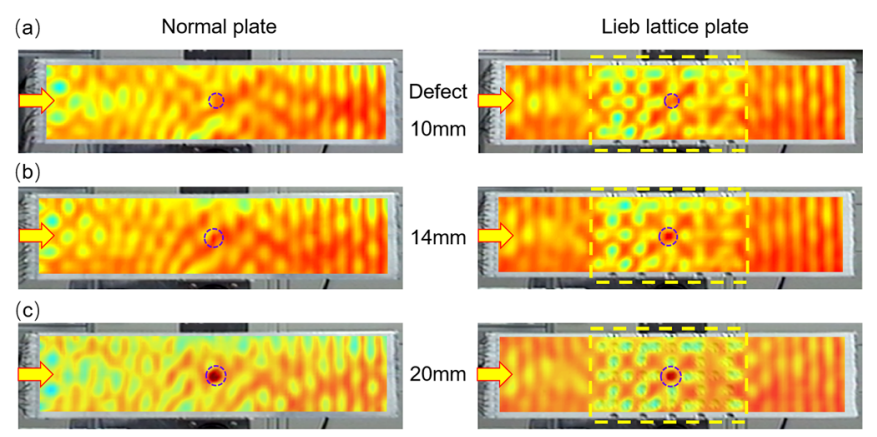

FIG. 5. Comparison of energy density distributions in a normal plate and a Lieb-like lattice waveguide under the same defect with diameters of (a) $10 \mathrm{~mm}$, (b) $14 \mathrm{~mm}$, and (c) $20 \mathrm{~mm}$.

The proposed Lieb-like lattice structure is impinged by an incident A0 planar wave at Dirac-like point I frequency $\left(f_{D 1}\right.$ $=6.27 \mathrm{kHz})$. The out-of-plane displacement field contour $\left(u_{z}\right)$ is captured in Fig. 3(a). An incident S0 planar wave at Dirac-like point II frequency $\left(f_{\mathrm{D} 2}=29.24 \mathrm{kHz}\right)$ is also stimulated, and the wave field distribution of in-plane displacement along the incident direction $\left(u_{x}\right)$ is shown in Fig. 3(b). The wave field contours indicate that there is no phase change when the relevant wave modes pass through the Lieb-like metamaterial slab, achieving an almost total transmission. The numerically transmittance coefficients around dual Dirac point frequencies are calculated and shown in Fig. 3(c). It is confirmed that nearly full transmission is observed for various wave modes around their Dirac points because of the zero-refractive index.

We further explore the applications of the proposed Lieblike metamaterial in shaping the wave-front pattern. We introduce a defect into the elastic waveguide. One defect is located before the Lieb-like lattice region [Figs. 3(d) and 3(e)], while the other defect location is within the Lieb-like region [Fig. 3(f)]. The input surface is stimulated by an incident A0 planar wave at the frequency of Dirac-like point I and a S0 wave at the frequency of Dirac-like point II, respectively. It is clearly observed that the wave-front is distorted significantly for both wave modes when the planar waves pass through the normal waveguide. However, if we set up a Lieb-like metamaterial region after the defect, the transmitted waves display a non-distorted planar wave-front pattern after passing through the metamaterial region. When the defect is located within the metamaterial region [Fig. 3(f)], we observe significant wave distortion in the normal plate, but when the defect is in the Lieb-like metamaterial plate, the output waves maintain a regular and non-distorted wave-front. The effect of damping on wave-front shaping performance of the Lieb-like lattice plate is further discussed in the supplementary material.

Experimental investigation is performed to validate the extraordinary transmission properties of the Lieb-like lattice waveguide. The experimental setup is shown in Fig. 4(a), and more details are introduced in the supplementary material. Compared to the numerical model, the thickness and the unit cell size of the fabricated Lieb-like lattice plate are scaled to half the original dimensions for better fabrication. The resulting frequencies for 
Dirac-like points I and II are doubled to $12.54 \mathrm{kHz}$ and $58.48 \mathrm{kHz}$, respectively. As shown in Fig. 4(b), the measured transmitted amplitude is nearly constant across the two Dirac-like cones and the near total transmission is obtained, which agrees with the numerical results (see the supplementary material).

To investigate the wave-front shaping capability, the outof-plane displacement contours of a normal plate and the Lieb-like lattice plate under A0 planar wave excitation are acquired. The same punched hole is introduced to the center of both the plates. Varying the defect size, the comparisons of the full-field wave propagation in the normal and the Lieb-like lattice waveguides are visualized (see the supplementary material), and one typical comparison is shown in Fig. 4(c). It can be observed that the disturbance effect on the wave-front is clearly captured in the normal plate, but the wave-front shape in the Lieb lattice plate still maintains an almost non-distorted planar pattern, which demonstrates the capabilities of wave-front shaping and reconstructing in the proposed Lieb-like metamaterial. We further capture the root mean square (RMS) of the transient responses to compare the energy density distribution during the same duration. The relevant results obtained under different defect sizes are shown in Fig. 5. It is illustrated that the defect location and the Lieb-like pattern can be clearly recognized. Compared to the disturbed and irregular waveforms in a normal plate, the robustness of wave-front shaping and reconstruction performances in the Lieb lattice waveguide is unambiguously verified.

In summary, we have proposed and demonstrated an elastic Lieb-like metamaterial that can simultaneously generate two Dirac-like cones around different Dirac points at the BZ center. We present theoretical, numerical, and experimental investigation that the proposed waveguide can be mapped to an effective medium with double-zero-index properties around the excitation frequencies of both Dirac-like cones. The zero-effectivedensity and infinite modulus for A0 and S0 modes waves have been realized. Near full transmission without phase variation has been verified. The ability of wave-front shaping is achieved for both $\mathrm{A} 0$ and $\mathrm{S} 0$ waves. The robust performance of this doublezero-index elastic waveguide under various defect sizes and damping has been discussed. This study extends the pathway for designing extraordinary elastic waveguide devices.

See the supplementary material for more discussion on the robustness and performance of the Lieb-like lattice waveguide.

K. T. Tan acknowledges the Faculty Start-Up Grant support from The University of Akron. J.C. gratefully acknowledges financial support from the MINECO through a Ramón y Cajal Grant (No. RYC-2015-17156). Z. Li acknowledges financial support from the National Natural Science Foundation of China under Grant No. 11672004.

\section{REFERENCES}

${ }^{1}$ T. Schwartz, G. Bartal, S. Fishman, and M. Segev, Nature 446, 52 (2007).

${ }^{2}$ S. Mukherjee and R. R. Thomson, Opt. Lett. 40, 5443 (2015).

${ }^{3}$ S. Longhi, M. Marangoni, M. Lobino, R. Ramponi, P. Laporta, E. Cianci, and

V. Foglietti, Phys. Rev. Lett. 96, 243901 (2006).
${ }^{4}$ R. Fleury, D. L. Sounas, C. F. Sieck, M. R. Haberman, and A. Alù, Science 343, 516 (2014).

${ }^{5}$ N. Boechler, G. Theocharis, and C. Daraio, Nat. Mater. 10, 665 (2011).

${ }^{6}$ Z. Liu, X. Zhang, Y. Mao, Y. Zhu, Z. Yang, C. T. Chan, and P. Sheng, Science 289, 1734 (2000).

${ }^{7}$ N. Kaina, F. Lemoult, M. Fink, and G. Lerosey, Nature 525, 77 (2015).

${ }^{8}$ B.-I. Popa, L. Zigoneanu, and S. A. Cummer, Phys. Rev. Lett. 106, 253901 (2011).

9J. Christensen, M. Willatzen, V. Velasco, and M.-H. Lu, Phys. Rev. Lett. 116, 207601 (2016).

${ }^{10}$ S. A. Cummer, J. Christensen, and A. Alù, Nat. Rev. Mater. 1, 16001 (2016).

"B. Liang, X. Guo, J. Tu, D. Zhang, and J. Cheng, Nat. Mater. 9, 989 (2010).

${ }^{12}$ B. Liang, B. Yuan, and J.-C. Cheng, Phys. Rev. Lett. 103, 104301 (2009).

${ }^{13}$ D. L. Sounas and A. Alù, Nat. Photonics 11, 774 (2017).

${ }^{14}$ G. C. Ma and P. Sheng, Sci. Adv. 2(16), e1501595 (2016).

${ }^{15}$ K. Bertoldi, V. Vitelli, J. Christensen, and M. van Hecke, Nat. Rev. Mater. 2(11), 17066 (2017).

${ }^{16}$ T. Neupert, L. Santos, C. Chamon, and C. Mudry, Phys. Rev. Lett. 106, 236804 (2011).

${ }^{17}$ E. Tang, J.-W. Mei, and X.-G. Wen, Phys. Rev. Lett. 106, 236802 (2011).

${ }^{18}$ C. Weeks and M. Franz, Phys. Rev. B 82, 085310 (2010).

${ }^{19}$ V. Iglovikov, F. Hébert, B. Grémaud, G. Batrouni, and R. Scalettar, Phys. Rev. B 90, 094506 (2014).

${ }^{20}$ L. Ge, Phys. Rev. A 92, 052103 (2015).

${ }^{21}$ S. Mukherjee, A. Spracklen, D. Choudhury, N. Goldman, P. Öhberg, E. Andersson, and R. R. Thomson, Phys. Rev. Lett. 114, 245504 (2015).

${ }^{22}$ D. Guzmán-Silva, C. Mejía-Cortés, M. Bandres, M. Rechtsman, S. Weimann, S. Nolte, M. Segev, A. Szameit, and R. Vicencio, New J. Phys. 16, 063061 (2014).

${ }^{23}$ J. Lu, C. Qiu, S. Xu, Y. Ye, M. Ke, and Z. Liu, Phys. Rev. B 89, 134302 (2014).

${ }^{24} \mathrm{X}$. Zhang and Z. Liu, Phys. Rev. Lett. 101, 264303 (2008).

${ }^{25}$ X. Huang, Y. Lai, Z. H. Hang, H. Zheng, and C. Chan, Nat. Mater. 10, 582 (2011).

${ }^{26}$ M. Dubois, C. Shi, X. Zhu, Y. Wang, and X. Zhang, Nat. Commun. 8, 14871 (2017).

${ }^{27}$ F. Liu, X. Huang, and C. Chan, Appl. Phys. Lett. 100, 071911 (2012).

${ }^{28}$ F. Liu, Y. Lai, X. Huang, and C. Chan, Phys. Rev. B 84, 224113 (2011).

${ }^{29}$ J. Mei, Y. Wu, C. T. Chan, and Z.-Q. Zhang, Phys. Rev. B 86, 035141 (2012).

${ }^{30} \mathrm{~S}$. Wu and J. Mei, AIP Adv. 6, 015204 (2016).

${ }^{31}$ Z.-G. Chen, X. Ni, Y. Wu, C. He, X.-C. Sun, L.-Y. Zheng, M.-H. Lu, and Y.-F. Chen, Sci. Rep. 4, 4613 (2014).

${ }^{32}$ Z. Zhang, Q. Wei, Y. Cheng, T. Zhang, D. Wu, and X. Liu, Phys. Rev. Lett. 118, 084303 (2017)

${ }^{33}$ Y. Li, Y. Wu, and J. Mei, Appl. Phys. Lett. 105, 014107 (2014).

${ }^{34}$ J. Mei, Z. Chen, and Y. Wu, Sci. Rep. 6, 32752 (2016)

${ }^{35}$ L. H. Wu and X. Hu, Phys. Rev. Lett. 114(5), 223901 (2015).

${ }^{36}$ P. Moitra, Y. Yang, Z. Anderson, I. I. Kravchenko, D. P. Briggs, and J. Valentine, Nat. Photonics 7, 791 (2013).

${ }^{37}$ Y. Li, S. Kita, P. Muñoz, O. Reshef, D. I. Vulis, M. Yin, M. Lončar, and E. Mazur, Nat. Photonics 9, 738 (2015).

${ }^{38}$ G. Ma, C. Fu, G. Wang, P. Del Hougne, J. Christensen, Y. Lai, and P. Sheng, Nat. Commun. 7, 13536 (2016).

39J. H. Oh, Y. E. Kwon, H. J. Lee, and Y. Y. Kim, Sci. Rep. 6, 23630 (2016).

${ }^{40}$ H. Zhu and F. Semperlotti, Phys. Rev. Appl. 8, 064031 (2017).

${ }^{41}$ Y. Lai, Y. Wu, P. Sheng, and Z.-Q. Zhang, Nat. Mater. 10, 620 (2011).

${ }^{42}$ F. Liu and Z. Liu, Phys. Rev. Lett. 115, 175502 (2015).

${ }^{43}$ F. Liu, F. Zhang, W. Wei, N. Hu, G. Deng, and Z. Wang, Phys. Rev. B 94, 224102 (2016).

${ }^{44}$ M. S. Dresselhaus, G. Dresselhaus, and A. Jorio, Group Theory: Application to the Physics of Condensed Matter (Springer Science \& Business Media, 2007).

${ }^{45}$ L. C. L. Y. Voon and M. Willatzen, The kp Method: Electronic Properties of Semiconductors (Springer Science \& Business Media, 2009).

${ }^{46}$ H. Chen, H. Nassar, and G. L. Huang, J. Mech. Phys. Solids 117, 22 (2018).

${ }^{47}$ Y. Chen, X. N. Liu, and G. K. Hu, J. Mech. Phys. Solids 122, 54 (2019). 